\title{
A Comparison of Oral and Intravenous Glutamine Therapy Effects On Mucositis Development in Acute Leukemic Patients After High Dose Chemotherapy Supplementation
}

\author{
A. Dilara Demir ${ }^{1 *}$ and Ilhami Kiki ${ }^{2}$ \\ ${ }^{1}$ Department of Internal Medicine, Amasya University Research Hospital, Amasya. Turkey. \\ ${ }^{2}$ Department of Haematology, Ataturk University Faculty of Medicine. Erzurum, Turkey.
}

Corresponding Author: A.Dilara Demir, Internal Medicine Department, Amasya University Research Hospital, 05000 Amasya, Turkey; Tel:+90 35821840 00; Fax: +90 35821200 01; E-mail: adilarabay@gmail.com

Received: 27 July 2017; Accepted: 12 December 2017; Published: 18 December 2017

\begin{abstract}
Aim: We compared the oral and intravenous glutamine therapy effects on mucositis development in acute leukemia patients after high dose chemotherapy supplementation. This research was applied in high dose chemotherapy patients in order to determine the effects of parenteral glutamine support on mucositis development. The results of oral and parenteral glutamine therapy are compared and analyzed for variations in mucositis formation.
\end{abstract}

Materials and Methods: The present study includes the results of glutamine therapy performed on two patient groups. A total of 70 individuals ( 33 female and 37 male) were included in the study. Out of these patients, 35 were given oral glutamine whereas the remaining 35 were given glutamine via an intravenous route following chemotherapy. Patients were between 16 and 71 years of age.

IV-therapy and oral therapy patients were selected as first and second groups, respectively. The same amount of Lalanyl Glutamine solution was given to both groups in equal time intervals. Mucositis grading was performed according to the criteria of the American Academy of Pediatric Dentistry and staging was carried out according to oral mucositis index.

Results: When records of both groups were analyzed seperately, mild to moderate mucositis was determined in $85.7 \%$ (30 of 35) of the patients whereas severe mucositis was observed in $14.3 \%$ (5 of 35) patients who received 
oral glutamine support. On the other hand, mild- to moderate mucositis developed in all 35 patients (100\%) who received intravenous glutamine support, but no severe mucositis developed in those patients.

Conclusion: The result of this work can be summarized as; neither oral nor parenteral glutamine therapy prevents mucosite development, but parenteral glutamine therapy has a preventative effect on the development of severe mucositis.

Keywords: Acute leukemia; Chemotherapy; Glutamine; Oral; Parenteral; Mucositis

\section{Introduction}

Although recent advances have been achieved in the therapy of malignant diseases, infections are still the most significant cause of mortality. Both normal healthy cells with high replication rates and malignant cells are negatively affected by heavy cancer therapy. This situation may result in very serious conditions which may cause changes from formation of oral mucositis to sepsis. In the case of hypercatabolic state and mucositis, glutamine is given as external supportive therapy. Glutamine contains the highest amount of free amino-acid in the body which is known to be the precursor of the glutathione as being one of the most significant antioxidants.

Dietary intake of glutamine and glutamate are the major sources of energy for enterocyte, when a proper diet is applied. However, these cells require serious amounts of glutamine in circulation when diet support ends. Similarly, when active tumors exist in the body, glutamine demand is provided by the glutamine pool of the body. Besides glutamine is a nucleotide synthesis precursor. It is a significant source of energy for rapidly dividing cells, such as the gastrointestinal system epithelium. Important substrates for lymphocytes and macrophages exist in critical cells of the immune system. Various studies show that glutamine rich total parenteral nutrition improves immunologic situations and diminishes hospitalization periods of patients in critical illness stages [1]. In the case of critical abstruse glutamine deficiency is within the plasma and tissues, strong correlation is found between diminished plasma glutamine level and increased mortality. Currently, there is strong evidence of successful usage of IVglutamine support in onchologic, heamatologic and in critically ill patient situations [2].

In a randomized double-blind placebo- controlled study, B.J.Morlion et al. [3] showed that following major abdominal operations, glutamine enriched total parenteral nutrition therapy raised the nitrogen balance, lymphocyte turnover, leukotrien excretion from neutrophils and shortened hospitalization time. Research by Ziegler et al. [4] on the efficiency of parenteral glutamine usage in patients with hematopoetic stem cell transplantation, shows that; although time doesn't decay until fever, the antibiotic requirement, neutrophile engraftment, and nitrogen balance result in a decrease in the frequency of clinical infection and hospitalization. Schloerb \& Amare [5] compared parenteral glutamine support with plasaebos in 29 patients after administering autologous or allogeneic stem cell transplantation. Although, hospitalization time was shortened in the group receiving glutamine therapy, a meaningful difference for the frequency of infection and mortality between two groups was not observed. 
However, in either of the two studies, no evidence was found that parenteral glutamine support prevents or diminishes mucositis development. Similarly, there exists conflicting evidence aboutefficiency of oral glutamine support. It is shown that oral glutamine is inadequate for preventing mucositis depending on 5-FU bolus infusion [6]. In research performed on 193 otolog and/or allogeneic stem cell transplantation patients, Anderson et al. [7] reported that oral glutamine support decreases oral pain as with the use of opiates, but is probably related to interaction with methotrexate. This efficiency is not observed in patients received allogeneic transplantation. In this work, the main purpose was to compare the effects of oral and parenteral glutamine therapy on mucositis development in patients who received high dose chemotherapy.

\section{Materials and Methods}

Seventy patients with acute leukemia, consisting of 37 males and 33 females referred to the Haematology Department of the Medical School Research Hospital at Ataturk University, have been accepted for this research. Patients range in age between 16 and 71 years. Patients receiving chemotherapy, but who do not have any metabolic disease and/or solid tumors, have been selected. Out of these patients, 35 were given oral glutamine whereas the rest (35) were given glutamine via an intravenous route following chemotherapy.

In induction treatment, prednisolone, vincristine, anthracyclines was given. The protocol called for adding early intensified treatments with cyclophosphamide, cytarabine and mercaptopurine. Intrathecal methotrexate was given for CNS prophylaxis. In consolidation therapy, medium- and high-dose methotrexate, mitoxantrone, and high-dose cytarabine was given. Maintenance therapy was done with methotrexate and 6- mercaptopurine. All of the patients were fed orally. Patients able to accept food, were fed by the fixed diet within hospital. No other nutritional support was given. Oral care was given with topical chlorhexidine and nystatin as a prophylactic. Every two days, all patients are orally examined to assess the severity of any mucositis.

The Statistical Software Package (SPSS) 21.0 packet program was used for data analysis. The categorical data were summarized by number and percentage. Numeric data were summarized with mean, standard deviation, minimum and maximum values. In the comparison of the between-group difference chi-square and Fisher's exact test was used. A margin of error for statistical significance was $<0.05$.

Patients were randomly divided into two groups. While $15 \mathrm{gm}$ glutamine was given orally to 35 patients in the first group, the same amount glutamine was given parenterally to 35 patients in the second group. In total, 70 patients were included in this study. Age, gender, illness,and chemotherapy protocol received for each patient was determined and recorded. On alternate days, oral examination was done for mucositis. This work has been a prospective, reconnaissance research effort. Permission has been obtained from Ataturk University Ethics Committee, before starting this study. The therapy procedure was explained in detail to patients,and both spoken and written approvals were obtained. In the first group, 48\% (17) of the patients had acute lenfoblastic leukemia (ALL) and 52\% (18) had acute myeloblastic leukemia (AML). In second group patient's 57\% (20 patients) was ALL and 43\% (15 patients) was 
AML. Mucose membrane, gingiva, tongue, teeth, saliva/xerostomia, swallow/chewing functions were examined on alternate days and recorded, while oral mucositis staging was performed.

In both groups, after the neutropeny period, L-alanyl glutamine solution was administered to the group that receive IV-therapy, and an equal amount of L-alanyl glutamine was administered to the group that received oral therapy. All were acute leucemic patients and received standard or high dose chemotherapy. On alternate days patients were examined for oral mucositis. Mucositis grading was performed according to the criteria of the American Academy of Pediatric Dentistry (Table 1) [8]. Mucositis staging was prepared according to oral mucositis indexes (Table 2) [9]. Careful observations were performed individually according to the criteria stated above.

\begin{tabular}{|l|}
\hline Mucosa membrane/ Gingiva \\
\hline (0) pink, wet, intense gingiva \\
\hline (1) red, edema, plaque \\
\hline (2) ulceration / bleeding \\
\hline tongue \\
\hline (0) pink and wet \\
\hline (1) rosted \\
\hline (2) raised / striated \\
\hline teeth \\
\hline (0) clean, no debris \\
\hline (1) less debris \\
\hline (2) debris \\
\hline (3) decayed \\
\hline saliva / xerostomia \\
\hline (0) wet \\
\hline (1) thick and/or viscouse \\
\hline (2) non / cotton mouth \\
\hline swallowing / chewing \\
\hline (0) normal \\
\hline (1)normal food can not be swallowed \\
\hline (2) soft food can not be swallowed \\
\hline (3) drink (liquid) can not be swallowed \\
\hline (4) mucosa can not be swallowed \\
\hline
\end{tabular}

Table 1: Mucositis Grading Based on AAPD Criteria (Am. Acad. of Pediatric Dentistry). 


\begin{tabular}{|l|}
\hline Oral Mucositis Indexes (62) \\
\hline Grade 0: no changes \\
\hline Grade 1: erythema and pain \\
\hline Grade 2: erythema+ ulcerous+ can consume solid \\
\hline Grade 3: ulcerous + can consume only liquid food \\
\hline Grade 4: can't be fed \\
\hline
\end{tabular}

Table 2: Index of Oral Mucositis

\section{Results}

Mild mucositis developed in $65(92.9 \%)$ and severe mucositis in 5 (7.1\%) of the individuals (a total of 70 patients). Mild mucositis (grade 0, 1, and 2) developed in all 35 (100\%) of the patients who were administered IV-therapy, but severe mucositis developed in non of the patients. Mild mucositis developed in $30(85.7 \%)$ of 35 patients to whom oral glutamine therapy was administered, whereas severe mucositis (grade 3 and 4) developed in only 5 (14.3\%) of them. This is statistically meaningful with $(\mathrm{p}<0.05)$.

In the IV-therapy group, severe mucositis did not develop, which is statistically limited in its meaningfulness when $p=$ 0.05 accepted. A larger study involving more patients would be required to achieve a statistically meaningful sample.

In $51(94.4 \%)$ patients mild mucositis developed, whereas severe mucositis developed in $3(5.6 \%)$ out of a total of 54 patients who received intensive therapy. Mild mucositis developed in 14 patients (87.5\%) and severe mucositis developed in 2 patients $(12.5 \%)$ out of 16 who received standard therapy. This result is not statisticaally meaningful $(\mathrm{p}=0.321)$ and reliable, since a $\mathrm{p}$-value much higher than 0.05 is needed.

Patients who are selected for this study have mean average ages defined as $36.8 \pm 17.2$ years (16-71). The entire group of 70 patients consisted of 37 males (52,9\% cumulative percent was $52.9 \%)$ and 33 females (47.1\% cumulative percent was $100.0 \%$ ). The mean average age of patients who received intravenous glutamine support was 34.8 \pm 16.5 and of the patients who received oral glutamine support $(38.8 \pm 17.8)$. Statisticaally there is no difference, between the two groups. The group that received oral glutamine was composed of 19 females (55\%) and 16 males $(45 \%)$.

In the group that received intravenous glutamine, 14 were female $(40 \%)$ and 21 were male $(60 \%)$. Of the patients who received oral glutamine support, 20 (47\%) were ALL, 15 (43\%) were AML. The patients who received IVglutamine support 17 (48\%) were ALL, and 18 of them (52\%) were AML. 
The patients selected for this study consisted of 70 individuals diagnosed for acute leukemia, but did not have solid organ tumors. 54 of patients' (77.1\%) received intensive chemotherapy (high dose cytosine arabinoside, high dose methotrexate), and 16 of them (22.9\%) received standard chemotherapy (Table 3). L-alanyl glutamine solution (15 gm 1x1) was administered to the group that received intravenous glutamine, and L-alanyl glutamine dipeptide (5gm $3 \times 1)$ therapy was administered to the group that received oral glutamine.Patients were divided into two groups as follows: oral glutamine support to 35 patients and intravenous glutamine support to 35 patients.

\begin{tabular}{|c|c|c|c|}
\hline $\begin{array}{c}\text { Glutamine } \\
\text { Support }\end{array}$ & $\begin{array}{c}\text { Therapy regimen: Intensive } \\
\text { therapy }\end{array}$ & $\begin{array}{c}\text { Therapy regimen: Standard } \\
\text { therapy }\end{array}$ & Total \\
\hline IV therapy & $26 ; 74.3 \%$ & $9 ; 25.7 \%$ & $35 ; 100.0 \%$ \\
\hline Oral therapy & $28 ; 80.0 \%$ & $7 ; 20.0 \%$ & $35 ; 100.0 \%$ \\
\hline Total & $54 ; 77.1 \%$ & $16 ; 22.9 \%$ & $7 ; 100.0 \%$ \\
\hline
\end{tabular}

Table 3: Therapy Regimen of the Intravenous and Oral Glutamine Support Groups.

In considering all of the patients, it is recorded that $65(92.9 \%)$ of them had mild-moderate grade mucositis (oral mucositis index of 1 and 2) and 5 (7.1\%) of them had severe mucositis (oral mucositis index of 3 and 4).

In comparison, patients who received intravenous glutamine support and oral glutamine support, it was determined that all of 35 patients (100\%) who received IV-glutamine support developed mild mucositis (grade 1 and 2 ) and no severe mucositis was $(0 \%)$ observed. Mild-to moderate mucositis developed in $30(85.7 \%)$ of 35 patients to whom oral glutamine support was administered, but severe mucositis (grade 3 and 4 ) developed in only 5 of them (14.3\%).

Comparisons were made according to the kind of chemotherapy received: intensive chemotherapy was administered to 54 patients, and standard chemotherapy was administered to 16 patients. Mild mucositis was found in 51 (94.4\%) of 54 patient' $\mathrm{s}$ who received intensive therapy and severe mucositis was found in $3(5.6 \%)$ of them. Mild mucositis was found in $14(87.5 \%)$ of patients who received standard therapy and severe mucositis was found in 2 of them (12.5\%). The Q-square value was 0.897 and $\mathrm{p}=0.321$. No logical relation was found between mucositis degree and therapy regime.

When the two groups are separately analyzed; mild-to moderate mucositis was observed in 30 patients (85.7\%) and severe mucositis was observed in 5 patients $(14.3 \%)$ out of the 35 who received oral glutamine support. On the other hand, mild-to moderate mucositis was observed in all 35 (100\%) patients who received intravenous glutamine support and no severe mucositis was observed in any of the patients (Table 4). 


\begin{tabular}{|c|c|c|c|c|}
\hline \multirow[b]{2}{*}{$\begin{array}{l}\text { Mode of } \\
\text { Support }\end{array}$} & \multicolumn{2}{|c|}{ Mild- moderate mucositis (grade 1-2) } & \multicolumn{2}{|c|}{ Severe mucositis (grade 3-4) } \\
\hline & eStandard Therapy & High dose Therapy & Standard Therapy & \begin{tabular}{|l|} 
High dose \\
Therapy
\end{tabular} \\
\hline oral glutamine support & $5 ; 71.4 \%$ & $25 ; 89.3 \%$ & $2 ; 28.6 \%$ & $3 ; 10.7 \%$ \\
\hline IV-glutamine support & $9 ; 100.0 \%$ & $26 ; 100.0 \%$ & 0 & 0 \\
\hline Total & $30 ; 85.7 \%$ & & $5 ; 14.3 \%$ & \\
\hline
\end{tabular}

Table 4: Index of Oral Mucositis. Oral and intravenous glutamine support received high dose and standard therapy groups.

Since the requirement of all expected frequency values obtained from Q-square calculations should be greater than 5 was not satisfied, the Fisher Exact Test was applied. A p-value of 0.256 was determined.

\section{Discussion}

Acute leukemia is a group of malignant illnesses developing after neoplastic transformation of haematopoietic stem cells and characterized by a defect of differentiation and maturation in leukemic cells. Hence, leukemic cells which have over reproduction ability invade bone marrow peripheral blood and other tissues [10]. Acute leukemia incidence is (0.8-1.8) /100,000 per year. The occurrence of acute leukemias' varies according to age. In general, ALL is seen more frequently in childhood while AML is seen more frequently in adults [11]. Mucositis which depends on chemotherapy is limited by personal identity, short duration, unexpected haematologic effect, and can influence all gastro-intestinal systems. Incidence and severity of oral mucositis is influenced by administered chemotherapeutic agents and factors related to the patient. Oral illness (bad oral hygiene, decayed teeth, periodontal illness) existing before therapy, may cause increased mucositis by chemotherapy.

Participants who were included in this study, consisted of 70 patients diagnosed with acute leukemia, but without any solid organ tumors. Fifty four patients (77.1\%) received intensive chemotherapy and 16 (22.9\%) of them received standard chemotherapy. It was determined that the Q-square value is 5.385 and p-value is 0.054 . Our study is assumed meaningful for results of $\mathrm{p}=0.6$ and of limited meaningfulness for $\mathrm{p}=0.5$. The degree of significance is low. This may be related to the patient sample size. Among the patients included in this study, mild mucositis has developed in $65(92.9 \%)$ and severe mucositis in 5 (7.1\%). By comparision of the results obtained from the intravenous therapy (IVT) and oral therapy (OT) patients, it was clearly observed that mild (grade 0, 1, and 2) mucositis developed in all 35 intravenous glutamine received patients $(100 \%)$, whereas no severe mucositis was noted in this group. The observed percentage of mucositis development in 35 patients who received oral glutamine therapy was found to be only mild mucositis in $30(85.7 \%)$ and severe mucositis in $5(14.3 \%)$ of the patients.

In this work, we did not search for a relationship between degree of mucositis and local infection, systemic bacteriemia, viremia, or fungemia. Accepting that glutamine plays a significant role in mucositis prophylaxis, the main purpose of this study is focused on finding out if any difference exists in preventive efficiency of usage of 
glutamine (oral or IV-parenteral) on mucositis development. The ultimate purpose was to prove the possibility of preventing mucositis development. Before mucositis development, all patients received oral and IV glutamine support together with topical chlorhexidine and nystatin as profilactic.

Oral care was applied to all patients, but newly diagnosed acute leukemia patients' teeth and oral therapy was delayed because of their poor general condition and risk of hemorrhage. Oral problems for patients in remission who had high dose chemotherapy administered, resolved before starting therapy. There is no significant difference between the two groups of patients in terms of oral hygiene $(p>0.05)$. Clinical and metabolic effects of parenteral glutamine solutions were studied in many sub-group patients, primarily in intensive care patients and those receiving surgery. Bart J. Morlion et al. [3] showed in the randomized double blind plasebo controlled study that, following a major abdominal operation, glutamine enriched total parenteral nutrition therapy increased nitrogen balance, lenfosit turnover, and leukotrien excretion from neutrophils and shortened hospitalization time. In various studies it is shown that glutamine support, especially usage of high dose parenteral glutamine diminishes infectious complication ratios, mortality ratio, hospitalization time in many patient groups like critically ill patients, patients with short bowel syndrome, travma patients, burns, patients with colorectal care, intensive care patients, inflammatory intestinal illnesses, and diminished death risk depends on intensive care- acquired infections (12 \& 13). Results obtained in patients that were administered oral and IV-glutamine support, have not been compared with complications caused by the applied therapy such as, neutropeny developing period, frequency of febrile neutropeny, and mortality ratio. Instead, these two groups of patients were compared for mucositis development ratio, mucositis severity, and appearance time after chemotherapy.

As a result, it was found in both groups that the mucositis development ratio is the same, but severe mucositis did not develop in the parenteral glutamine receiving group, and severe mucositis was detected in $14.3 \%$ of the oral glutamine- receiving patients. Consequently, it is concluded that in standard and high dose chemotherapy recipient patients, glutamine supplementation does not prevent mucositis formation, but parenteral glutamine supplementation has significant preventative effect on the formation of severe mucositis. Oral and parenteral glutamine formulas were used however there is debate about the efficacy of glutamine preparates for preventing mucositis development. In a work by Ziegler et al. [4], it is reported that in patients who received parenteral glutamine, although antibiotics were needed during the period of fever reduction, neutrophile engraftment does not shorten but results in better nitrogen balance, diminished frequency of clinical infection, and shortened hospitalization time. Schloerb and Amare [5], compare parenteral glutamine support with plasebo usage in 29 patients who underwent autologous or allogeneic stem cell transplantation. Although hospitalization time is shorter in the glutamine supplemented group significant and reliable differences could not be found between the two groups both in terms of frequency of infection and in mortality. However in both studies there is no evidence found about the preventative or weakening effect of parenteral glutamine support in mucositis formation. In fact, there is debate about the benefit of oral glutamine support. It has been shown that oral glutamine supplements are inadequate in controlling mucositis formation depending on 5-FU bolus infusion [6]. Schloerb and Skikne [14] showed as a result of study performed on 66 patients given autologous or allogeneic stem-cell transplantation, oral glutamine support according to plasebo does not 
reduce hospitalization time, number of total parenteral feedings per day, neutrophile improvment time, sepsis, mucositis formation and diarrhea, but can improve long term allogeneic transplantation.

There is no consensus on the efficacy of glutamine support for preventing oral mucositis in the studies stated above. The studies basically cover cases of otologous or allogenic transplantation, however in the present work, the majority of our cases consist of patients who received high dose chemotherapy and hence mucositis risk was too high, comparatively. Covaluation of our data (by comparing parenteral and oral glutamine support) clearly revealed the fact that parenteral glutamine support has much higher efficacy in preventing severe mucositis formation. A possible explanation of oral glutamine supplementation's weak efficacy is because of it's limited bio-availability on mucosal surfaces due to low glutamine solubility. Also oral intake deficiency due to chemotherapy and vomiting are factors limiting efficacy of oral glutamine.

For this reason, a new oral L-glutamine formulation is proposed to have high bioavailibility in oral mucosa. Analysis of 326 chest-cancer patients who received Antracyclin based chemotherapy reported that in spite of the presence of LGlutamine solution, mucositis development was not prevented, but it lightened the pain according to placebo [15].

Patients used a glutamine preparate that can be orally taken and with lesser bioavailibility. In this patient group, severe mucositis developed in five cases. On the other hand, the fact that no severe mucositis development occurred in patients who received parenteral glutamine support,it is concluded that the degree of mucositis is inversly proportional to the plasma glutamine level. Absorbtion deficiency caused by factors such as low bioavailibility, use of medicine, vomiting, and mucosal atrophy may diminish oral glutamine's efficacy. Previous research indicated that parenteral glutamine support increases plasma glutamine level more than oral glutamine support [16].

In a review prepared from randomized clinical trials between 1983 and 2013 by Marta Sandini et al, the effect of IV glutamine in patients undergoing elective major abdominal surgery was analyzed. 640 patients were given intravenous glutamine and 603 patients did not receive glutamine. As a result, they found that glutamine shorten the length of hospital stay without affecting the complication rate [17].

In a meta analysis, Bollhald L. et al. saw that parenteral glutamine support reduces the infections and length of hospital stay, but could not show a significant reduction in mortality in patients with severe disease [18].

In another review, Wischmayer et al has emphasized parenteral glutamine with the support of nutrition to shorten the length of stay and hospital mortality in critically ill patients, and the need to continue as a component of nutritional support [19].

In a study, Ricardo Garib et al. evaluated the inflammatory and survival responses after parenteral glutamine infusion in experimental acute pancreatitis. They suggest that a high dose of parenteral glutamine protects against stress-induced organ damage by improving cytokine profiles [20]. 
Heyland and friends compared glutamine alone, antioxidants alone, and glutamine + antioxidants on mortality, in 1223 mechanically ventilated adult patients with multiorgan failure. They suggested that high dose glutamine was not beneficial and may be associated with increased mortality in critically ill patients with multiorgan failure[21].

In a study Hülya Sungurtekin and friends compared the effectiveness of enteral, parenteral and combined enteralparenteral glutamine supplementations in critically ill patients. Mortality was found same between groups. But in parenteral glutamine administrated group had less stay of intensive care unit [22].

Sezin Yuce Sari and friends studied the effect of glutamine and arginine-enriched nutritional support in cancer patients treated with intensity-modulated radiotherapy. They studied 29 patients. They suggested social functions, pain, appetite, dry mouth, sticky, saliva , trouble with taste, social eating, and swallowing problems, were significantly worse in the control group than glutamine-arginine received group [23].

Consequently, by this study it is shown that both oral and parenteral glutamine support do not prevent mucositis development, but parenteral glutamine support does prevent further development of severe mucositis. Randomized research to be performed on the larger patient sample is proposed to obtain more statistically significant information about this subject, which will provide application of covariance statistics.

More accurate results can be obtained by monitoring a large number of patients while using a control group in correlation with observed values.

\section{Conclusion}

We aimed to compare the oral and intravenous glutamine therapy effects on mucositis development in acute leukemic patients after high dose chemotherapy supplementation. We can summarize that neither oral nor parenteral glutamine therapy prevents mucosite development, but parenteral glutamine therapy has a profilactic effect on the development of severe mucositis. We believe that studies which include a larger patient sample and control group would be useful.

\section{Conflict of Interest}

None to declare.

\section{Acknowledgement}

Authors are grateful to Dr. Kadriye Tanas and Dr. Hilal Alpcan for technical help and Prof. Dr. Mehmet Gundogdu, Department Chair of Interial Diseases, Faculty of Medicine, Ataturk University, for providing the facilities to this study.

\section{References}

1. Mondello S, Italiano D, Giacobbe MS, Mondello P, et al. Glutamine Supplemented Total Parenteral 
Nutrition Improves Immunological Status In Anorectic Patients. Nutrition 677 (2010): 681.

2. Wernerman J. Clinical Use of Glutamine Supplementation. J Nutr 138 (2008): 2040-2044.

3. Morlion BJ, Stehle P, Wachtler P, Siedhoff H, Köller M, et al. Total Parenteral Nutrition With Glutamine Dipeptide After Major Abdominal Surgery: A Randomized, Double-Blind, Controlled Study. Ann Surg 227 (1998): 302-308.

4. Ziegler TR, Young LS, Benfell K, Scheltinga M, Hortos K, et al. Clinical and Metabolic Efficacy of Glutamine Supplemented Parenteral Nutrition After Bone Marrow Transplantation: A randomized, doubleblind, controlled study. Ann Intern Med 116 (1992): 821-828.

5. Schloerb PR, Amare M. Total Parenteral Nutrition With Glutamine In Bone Marrow Transplantation and Other Clinical Applications: A randomized, double blind study. JPEN J Parenter Enteral Nutr 17 (1993): 407- 413 .

6. Okuno SH, Woodhouse CO, Loprinzi CL, Sloan JA, LaVasseur BI, et al. Phase III Controlled Evaluation of Glutamine For Decreasing Stomatitis in Patients Receiving Fluorouracil (5-FU)-based Chemotherapy. Am J Clin Oncol 22 (1992): 258-261.

7. Anderson PM, Ramsay NK, Shu XO, Rydholm N, Rogosheske J, et al. Effect of Low-dose Oral Glutamine on Painful Stomatitis During Bone Marrow Transplantation. Bone Marrow Transplant 22 (1998): 339-341.

8. Wilkes JD, et al. Council on clinical affairs. Clinical Guideline on The Dental Management of Pediatric Patients Receiving Chemotherapy, Bone Marrow Transplantation and/or Radiation. American Academy of Pediatric Dentistry 5 (2001): 82-84.

9. Kowanko I, Hodgkinson B. Prevention and Treatment of Oral Mucositis in Cancer Patients. Best Practice. 3 (1998): 1-6.

10. Lichtman MA. Acute Myelogenous Leucemia. In: Beutler E, Lichtman MA, Coller BC, Kipps TJ (eds). Williams Haematology, 6th edition. New York: McGraw-Hill 272 (1995): 298.

11. Champlin R, Golde DW. The leucemias In: Braunwald E, Isselbacher K.J, Petersdorf RG (eds). Harrison's Principles of Internal Medicine, 11th Edition. Philedelphia: Saunders, 1991:1552-1561.

12. Scolapio JS, McGreevy K, Tennyson GS, Burnett OL. Effect of Glutamine In Short-Bowel Syndrome. Clin.Nutr 20 (2001): 319-323.

13. Novak F, Heyland DK, Avenell A, Drover JW, Su X, et al. Glutamine Supplementation In Serious Illness: A Systematic Review of The Evidence. Critical Care Medicine 30 (2002): 2022-2029.

14. Schloerb PR, Skikne BS. Oral and Parenteral Glutamine In Bone Marrow Transplantation: A Randomized, Double-blind Study. JPEN J Parenter Enteral Nutr 23 (1999):117-122.

15. Peterson DE, Jones JB, Petit RG. Randomized, placebo-controlled trial of Saforis for prevention and treatment of oral mucositis in breast cancer patients receiving anthracycline-based chemotherapy. Cancer 109 (2007): 322-331.

16. Luo M, Bazargan N, Griffith DP, Estivariz CF, Leader LM, et al. Metabolic Effects of Enteral Versus Parenteral Alanyl-Glutamine Dipeptide Administration In Critically Ill Patients Receiving Enteral Feeding: A Pilot Study. Clin Nutr 27 (2008): 297-306.

17. Sandini M, Nespoli L, Oldani M, Bernasconi DP, Gianotti L, et al. Effect of Glutamine Dipeptide 
Supplementation on Primary Outcomes for Elective Major Surgery: Systematic Review and Meta-Analysis. Nutrients 7 (2015): 481-499.

18. Bollhalder L, Pfeil AM, Tomonaga Y, Schwenkglenks M. A Systematic Literature Review and Metaanalysis of Randomized Clinical Trials of Parenteral Glutamine Supplementation. ClinNutr 32 (2013): 213223.

19. Wischmeyer PE, Dhaliwal R, McCall M, Ziegler TR, Heyland DK, et al. Parenteral Glutamine Supplementation In Critical Illness: A Systematic Review. Crit Care 18 (2014): R76.

20. Garib R, Garla P, Torrinhas RS, Moretti AIS, Machado MCC, et al. Effect of Previous High Glutamine Infusion on Inflammatory Mediators and Mortality in an Acute Pancreatitis Model. Mediators Inflamm 2016: 7-8.

21. Heyland D.K, Elke .G,Cook D,Berger M., Wischmeyer. P.E,Albert .M et al. Glutamine and Antioxidants in the Critically Ill Patient: A Post Hoc Analysis of a Large-Scale Randomized Trial. Journal of Parenteral and Enteral Nutrition. Volume XX Number X Month 201X 1-9.

22. Sungurtekin H, Öztürk İ, Beder B, Daldal H, SerinS. Effect Of Glutamine Supplemented Nutrition via Different Routes on Mortality And Morbidity For Critically Ill Patients. Nobel Medicus 32 C ilt: 11, Sayı: 2

23. Sari S.Y, Yazici G, Yuce D, Karabulut E, Cengiz M, Ozyigit G. The effect of glutamine and arginine-enriched nutritional support on quality of life in head and neck cancer patients treated with IMRT. Clinical Nutrition ESPEN 16 (2016) 30e35

This article is an open access article distributed under the terms and conditions of the Creative Commons Attribution (CC-BY) license 4.0 